\title{
Pathologies
}

\section{de la muqueuse buccale chez le sujet âgé en perte d'autonomie}

\section{Diseases of the oral mucosa for the elderly person with diminishing autonomy}

\section{Résumé}

\section{MOTS-CLEFS :}

- Gériatrie, dépendance, dermatologie buccale.

\section{KEYWORDS:}

- Geriatrics, dependence, oral dermatology.
Le but de cet article est de présenter les lésions de la muqueuse buccale les plus fréquemment observées chez la personne âgée en situation de dépendance, en insistant sur l'importance capitale de leur détection précoce compte tenu des risques d'altération de l'état général de ces patients fragilisés. De plus, certaines de ces lésions peuvent avoir une évolution pouvant engager le pronostic vital en cas de diagnostic tardif.

\author{
Abstract
}

AOS 2013;262:13-23

DOI: $10.1051 / \mathrm{aos} / 2013204$

(C) EDP Sciences 2013

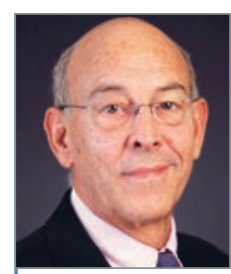

Didier GAUZERAN, Praticien hospitalier

chef de service honoraire des Hôpitaux de Paris,

Chargé de cours à l'Université de Paris - René Descartes,

Membre titulaire de l'Académie nationale de chirurgie dentaire,

Clinique Lambert-67, avenue Foch, 92250 La Garenne-Colombes.

Bernard SARICASSAPIAN, Praticien hospitalier du centre hospitalier de Guéret, Spécialiste qualifié en médecine bucco-dentaire, 39, avenue de la Sénatorerie, 23000 Guéret.

\section{INTRODUCTION}

L'odontologiste hospitalier est amené à voir en consultation des personnes âgées dépendantes, le plus souvent dans le cadre de l'urgence, à la demande du personnel soignant d'un EHPAD, du médecin traitant ou du chirurgien-dentiste de ville.

Il peut également être sollicité pour un malade hospitalisé dans un service de médecine gériatrique, de soins de suites et de réadaptation...
Les motifs de consultation sont, généralement, la recherche de foyers infectieux d'origine buccodentaire suite à la survenue de symptômes locorégionaux ou généraux dont l'étiologie n'est pas décelée, mais aussi des troubles du comportement non expliqués, des signes de dénutrition, des douleurs buccales exprimées ou supposées, des prothèses dentaires défectueuses...

Il s'agit le plus souvent d'un malade très " fragilisé " présentant de multiples comorbidités et dont la prise 
en charge requiert un plateau technique sécurisant et adapté.

L'état général du patient dépendant présente souvent de multiples pathologies. Son état est parfois aggravé par une maladie neurodégénérative et une malnutrition.

Pour la plupart des auteurs, un sujet âgé est une personne de plus de 75 ans ou de plus de 65 ans et présentant plusieurs pathologies [1]. L'examen clinique, de réalisation parfois difficile, met généralement en évidence une hygiène buccodentaire déficiente voire inexistante, un état dentaire et parodontal très mauvais, et la présence fréquente de lésions de la muqueuse [2] (fig 1).

Un rapport de la DGS (2006) va dans ce sens : « Les pathologies muqueuses, ulcérations, lésions traumatiques, candidoses sont fréquentes car favorisées par une hygiène insuffisante, une diminution de la sécrétion salivaire, le port de prothèses défectueuses, l'antibiothérapie au long cours, un diabète mal équilibré. » [3].

Une étude [4] réalisée en 2000 dans le département de l'Essonne portant sur « l'État de santé bucco-dentaire des personnes âgées dépendantes » précise que sur 308 des sujets examinés, $6 \%$ présentaient une pathologie de la muqueuse et $2 \%$ une ulcération traumatique.

Les lésions de la muqueuse sont la plupart du temps méconnues et doivent être systématiquement recherchées.

Elles sont favorisées par de nombreux facteurs dont, naturellement, l'action du vieillissement sur les tissus. Mais d'autres facteurs induisent parfois ces lésions : certaines pathologies générales, certains médicaments, la dépendance physique, l'état psychique, la dénutrition, le port de prothèses dentaires inadaptées [5]...

Le but de cet article est de présenter les lésions les plus fréquemment observées chez la personne âgée en situation de dépendance, en insistant sur l'importance capitale de leur détection précoce.

\section{CONSÉQUENCES DU VIEILLISSEMENT SUR LA SPHĖRE ORALE}

\section{Conséquences sur la muqueuse buccale}

La muqueuse buccale est constituée d'un épithélium malphigien, d'un chorion et, parfois, selon la topographie, d'une sous-muqueuse adipeuse.

Avec l'âge, ces tissus sont le siège de modifications histologiques :

I atrophie de l'épithélium avec disparition des crêtes épithéliales ;

I diminution du renouvellement cellulaire ;

1 diminution du nombre de cellules de Langerhans (rôle dans la réponse immunitaire);

I perturbation de la kératinisation témoignant d'un trouble dans la maturation des cellules épithéliales ;

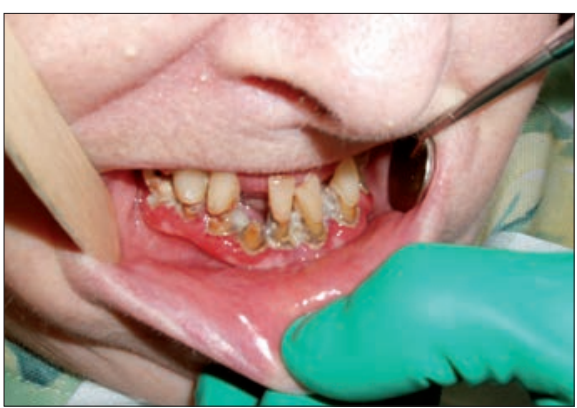

$<$ Fig. 1 :

État bucco-dentaire

d'une personne âgée en perte d'autonomie (coll. Saricassapian).

I au niveau du tissu conjonctif ou chorion, on note une diminution de la population cellulaire, une accumulation des fibres de collagène, une perte de l'élasticité par diminution des fibres élastiques, une altération des protéines structurelles...

I vascularisation déficiente par augmentation de l'athéromatose et de l'artériosclérose.

\section{Conséquences sur les glandes salivaires}

La sénescence entraîne une atrophie parenchymateuse progressive à partir de 50 ans. Cette atrophie physiologique peut atteindre $50 \%$ de la masse fonctionnelle chez les sujets de plus de 70 ans.

Une infiltration adipeuse se substitue aux acinis, une sclérose péricanalaire et une fibrose du tissu conjonctif de soutien complètent le tableau. Toutefois, ces perturbations ne sont pas suffisantes pour induire une xérostomie. Il reste en effet une réserve fonctionnelle parenchymateuse d'au moins 40 à $50 \%$.

La salivation au repos est diminuée mais la stimulation (alimentaire par exemple) conduit à une salivation conservée. Cependant, on constate chez ces patients un certain degré d'hyposialie et une augmentation de la viscosité de la salive. De plus, le moindre événement (médication sialoprive, maladies systémiques...) qui se surajoute à ces perturbations histologiques va déborder le potentiel fonctionnel résiduel et entraîner une sécheresse buccale [6].

\section{Conséquences de la sécheresse buccale}

La grande majorité des patients âgés souffrent d'une hyposialie plus ou moins marquée.

Rappelons que les principales causes de la sécheresse buccale sont : le syndrome de Gougerot-Sjögren, la radiothérapie cervico-faciale et surtout les médications sialoprives qui représentent environ $80 \%$ des causes d'hyposialie. Selon les études épidémiologiques, la population âgée consommerait entre 5 et 7 médicaments par jour $[1,7]$.

On dénombre environ 400 médicaments à effets sialoprives dont les psychotropes qui induisent les effets les plus marqués. Ces derniers peuvent induire une sclérose péricanalaire, parfois irréversible, avec diminution du flux salivaire sur une longue période. L'hyposialie entraîne une baisse du PH salivaire (acidification du milieu buccal) qui conduit à un déséquilibre de la flore buccale (développement des candidas, des streptocoques acidophiles...), une diminution des 
immunoglobulines salivaires qui favorise les infections, une diminution des enzymes à visée digestive, une diminution du rôle lubrifiant de la salive...

Par conséquent, l'hyposialie favorise les infections fungiques (candidoses) et parodontales, les polycaries (plaque dentaire acide), les langues lisses et vernissées (dépapillation), les mucites (inflammation des muqueuses) et facilite la survenue d'érosions d'origine traumatique. Il est aussi observé des dysphagies et des dysgueusies qui aggravent la malnutrition fréquente des sujets âgés.

À ces altérations des tissus buccaux que nous venons d'évoquer, s'ajoutent une diminution de la réponse immunitaire, une déshydratation et une dénutrition fréquentes, une iatrogénicité médicamenteuse qui occasionnent une fragilité des muqueuses, une diminution de la résistance tissulaire, un processus de réparation plus long impliquant une cicatrisation perturbée, le passage des lésions à la chronicité et un terrain favorable au développement de lésions précancéreuses et cancéreuses.

\section{INFECTIONS OPPORTUNISTES $[5,8]$}

\section{Candidoses}

Elles constituent certainement la pathologie la plus fréquemment observée chez la personne âgée.

Le Candida, albicans en particulier, est un endo-saprophyte habituel du tractus oro-gastro-intestinal.

La levure est en bon équilibre avec la flore commensale locale. Dans la cavité buccale, ils sont retrouvés à hauteur de 50 à $90 \%$ chez l'adulte âgé.

Les candidoses sont des infections opportunistes qui profitent des failles et des faiblesses de l'organisme et accompagnent alors souvent un diabète, un déficit salivaire (par diminution du PH buccal), un cancer, une séropositivité VIH... Plus simplement, elles peuvent suivre un traitement par antibiotique, corticoïde, neuroleptique...

Elles sont de plus favorisées par une hygiène buccale négligée voire absente. En effet, certaines circonstances locales et/ou générales vont engendrer un déséquilibre entre l'hôte et le Candida et, par conséquent, créer un milieu favorable à la colonisation, c'est-à-dire à la multiplication des Candidas sous forme de spores non pathogènes. Puis les Candidas vont quitter l'état lévurique pour passer à l'état filamenteux pathogène : c'est l'infection candidosique [9].

Les formes aiguës diffuses sont assez rares et touchent surtout, hormis le jeune enfant, le vieillard et l'adulte débilité (en particulier, lors d'une infection à HIV). Elles se présentent sous la forme d'efflorescences blanchâtres plus ou moins florides et qui se détachent facilement par simple raclage, laissant apparaître une muqueuse érosive (fig 2).

On peut rencontrer des formes érythémateuses (fig 3) où l'enduit blanchâtre est peu ou pas présent, à l'inverse des formes pseudomembraneuses.

Il existe aussi des formes localisées, essentiellement sur la langue et la voûte palatine, d'aspect érythémateux ou érythémato-pultacé.

Mais ce sont les formes chroniques en foyers qui dominent chez le sujet âgé : la candidose sous-prothétique, la glossite candidosique pseudomembraneuse ou érythémateuse et atrophique, la glossite médiane dite losangique ou rhomboïde, l'ouranite médiane, la chéilite commissurale candidosique qui est souvent associée à une autre localisation candidosique buccale (fig. 4).

Insistons sur la candidose sous-prothétique qui est très fréquente chez le patient âgé, au vu de la prévalence des prothèses amovibles chez ces sujets. Elle siège
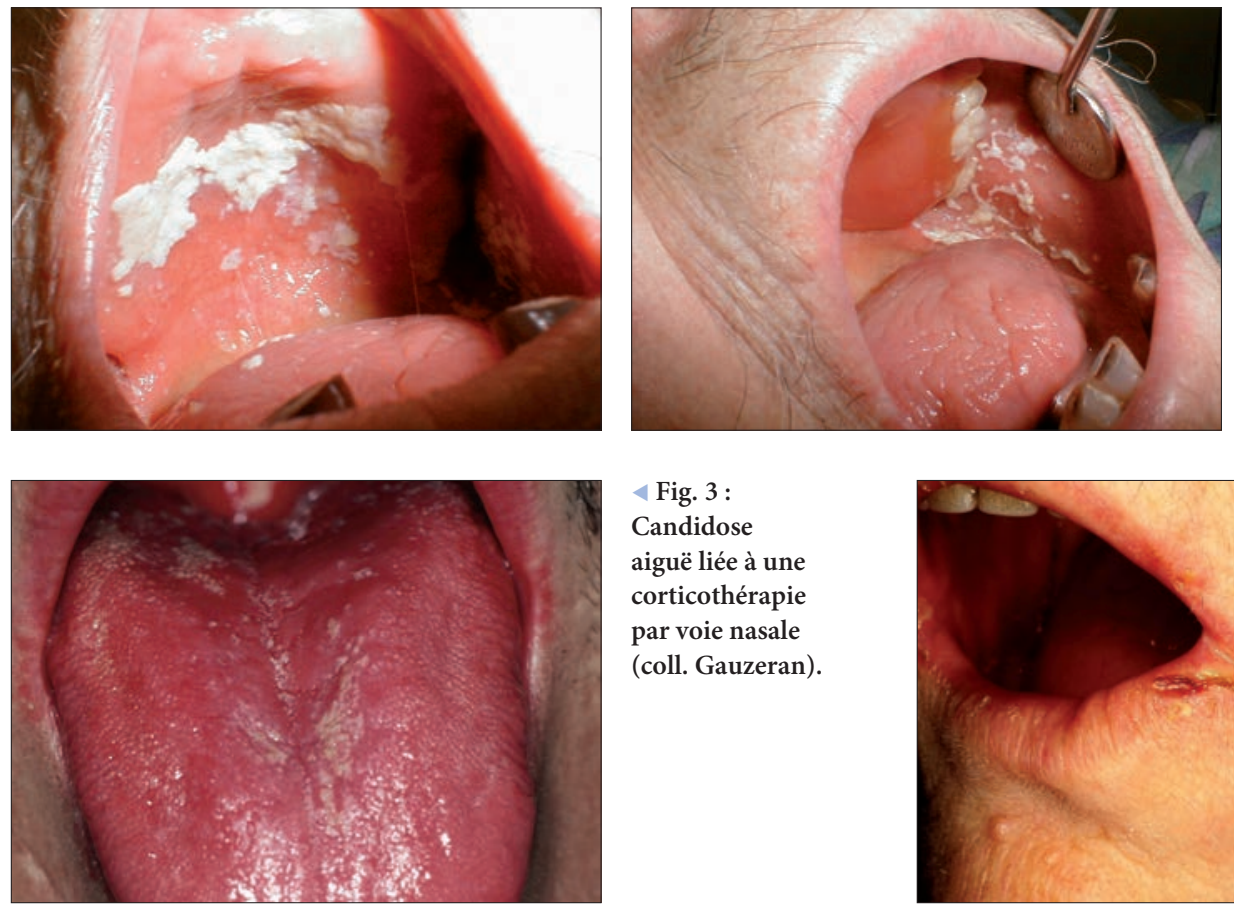

4 Fig. 3 :

Candidose aiguë liée à une corticothérapie par voie nasale (coll. Gauzeran).

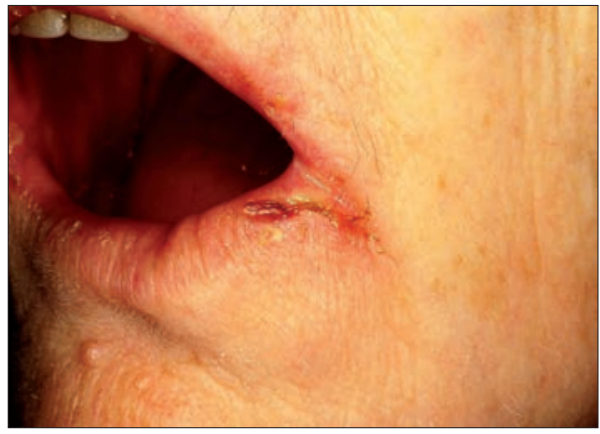

$\triangleleft$ Fig. 4 :

Chéilite

candidosique (coll. Gauzeran). 


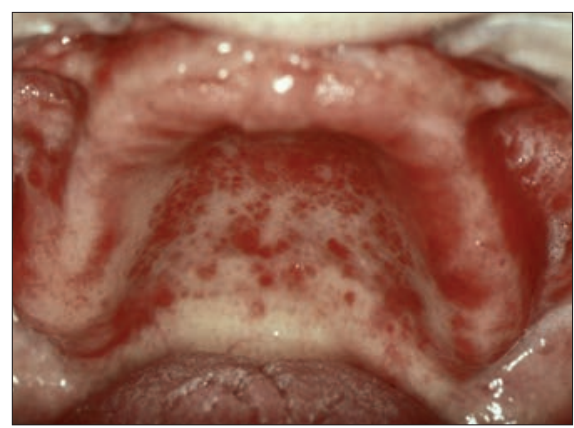

$\triangle$ Fig. 5 :

Candidose sous-prothétique (coll. Pompignoli).

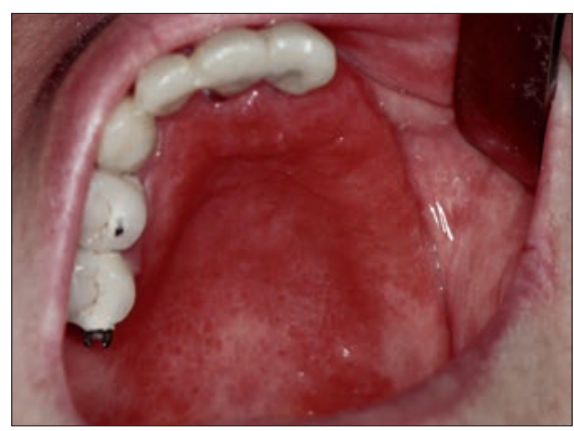

$\triangle$ Fig. 6 :

Candidose sous-prothétique (coll. Gauzeran).

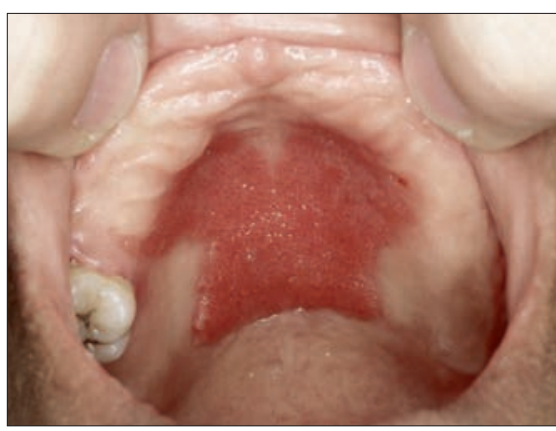

$\triangle$ Fig. 7 :

Hyperplasie épithéliale candidosique sous-prothétique (coll. Gauzeran). surtout au maxillaire et représente une inflammation chronique de la muqueuse palatine, en partie ou en totalité. Elle évolue selon trois stades :

| ouranite ponctuée : inflammation partielle de la muqueuse palatine qui présente parfois de petites pétéchies (fig 5);

| ouranite diffuse : inflammation palatine généralisée avec suffusion hémorragique fréquente (fig 6) ;

\ hyperplasie papillaire ou hyperplasie épithéliale inflammatoire : la lésion, qui épouse l'intrados de la prothèse, présente un aspect granuleux par la présence de tout petits nodules exophytiques inflammatoires, véritable arborescence fibro-épithéliale d'aspect mûriforme (fig 7).

Parfois, un examen mycologique s'avérera nécessaire afin de confirmer le diagnostic. Dans certaines situations, une biopsie, permettant un examen anatomopathologique, sera pratiquée, en particulier dans certaines formes chroniques.

Les formes aiguës répondent à un traitement médical au moyen d'antifongiques topiques ou systémiques selon le contexte, alors que certaines formes chroniques répondent plus souvent à une solution chirurgicale, avec contrôle anatomopathologique.

Le traitement doit d'abord être, si possible, étiologique (agir sur l'hyposialie, sur les médications sialoprives, sur l'équilibre glycémique en cas de diabète...).

Dans tous les cas, des soins locaux appropriés devront être mis en œuvre : hygiène bucco-dentaire, alcalinisation du milieu buccal, bains de bouche à base de chlorhexidine (action antifongique), suppression des prothèses amovibles pendant quelques jours, désin- fection soigneuse des prothèses, éviction des aliments acides, des sucres entre les repas...

\section{Viroses $[8,10]$}

Plus rares, mais non exceptionnelles chez les personnes âgées immunodéprimées, sont les atteintes aiguës herpétiques (Herpes virus) et le zona (Herpes zoster), redoutable par ses complications (algies post-zoostériennes). Ces affections contagieuses de l'adulte et du vieillard nécessitent une prise en charge en milieu hospitalier. Les précautions d'usage devront être prises lors de l'examen clinique, compte tenu des risques de transmission virale (fig 8).

\section{PATHOLOGIES LIÉES AUX PORT DE PROTHĖSES DENTAIRES $[8,10,11]$}

\section{Stomatite sous-prothétique [5]}

Elle peut prendre la forme d'une papillomatose inflammatoire qui, si elle est souvent d'origine candidosique (voir plus haut), peut s'avérer être liée à une irritation purement mécanique (fig 9).

Ceci étant, elle peut être colonisée secondairement par les Candidas.

Elle est en rapport avec un déficit d'hygiène généralement associé à l'inadaptation des prothèses qui engendre des microtraumatismes répétés, en particulier lors de problèmes neurologiques ou de traitement par neuroleptiques («dyskinésie du dentier») [12].

$\checkmark$ Fig. 8 a à c :

Zona du nerf maxillaire gauche : présence de vésicules sur la muqueuse de l’hémi-palais, de l’hémi-voile, de la joue et de la lèvre supérieure (coll. Saricassapian).
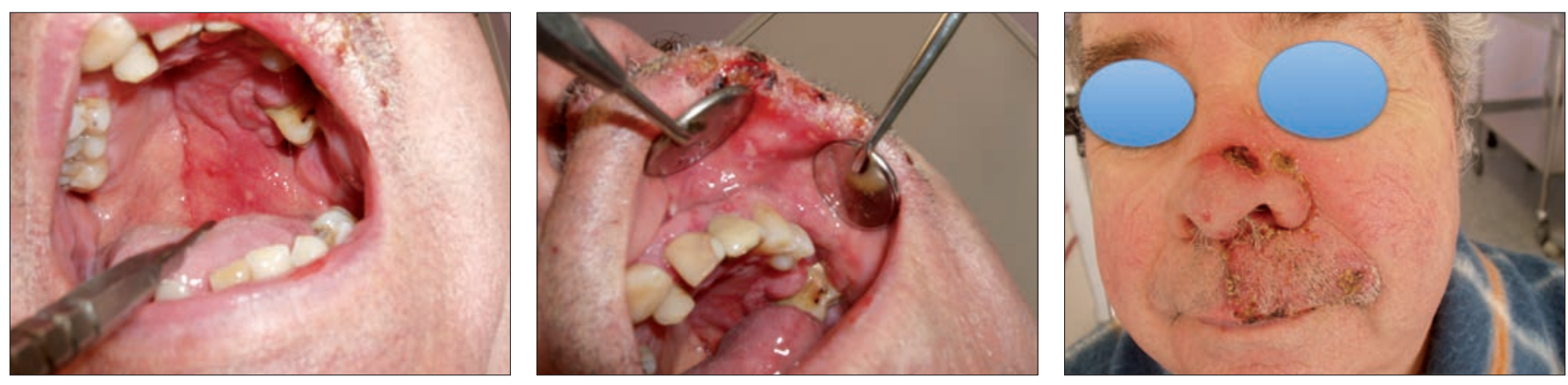


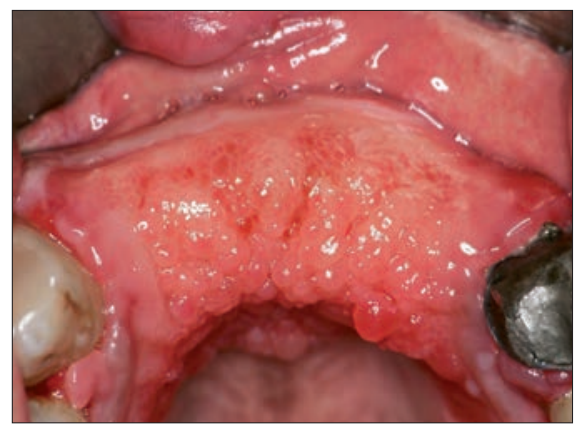

$\triangle$ Fig. 9 :

Papillomatose par traumatisme prothétique (coll. Gauzeran).

\section{Hyperplasie fibro-épithéliale $[5,8]$}

Il s'agit d'une lésion fréquente chez le sujet porteur de prothèses amovibles anciennes et inadaptées en raison de la résorption osseuse liée au vieillissement. Cela entraîne des mouvements répétés d'aspiration et de succion à l'origine d'une évagination de la muqueuse, réalisant une hyperplasie fibreuse sous la forme de bourrelets parfois en forme de «feuillets de livre». Les localisations sont le plus souvent situées au niveau du vestibule ou du sillon pelvi-lingual antérieur.

L'excision chirurgicale avec contrôle histopathologique et la réadaptation des prothèses seront la conduite à tenir (fig 10).

\section{Chéilite commissurale} (perlèche, chéilite angulaire) [5]

Il s'agit d'une infection fungique favorisée, entre autres, soit par l'affaissement physiologique de la commissure labiale (fonte musculaire du sujet âgé), soit par la diminution de la dimension verticale d'occlusion en rapport avec des prothèses anciennes et usées, et le manque de soutien tissulaire (fig 11).

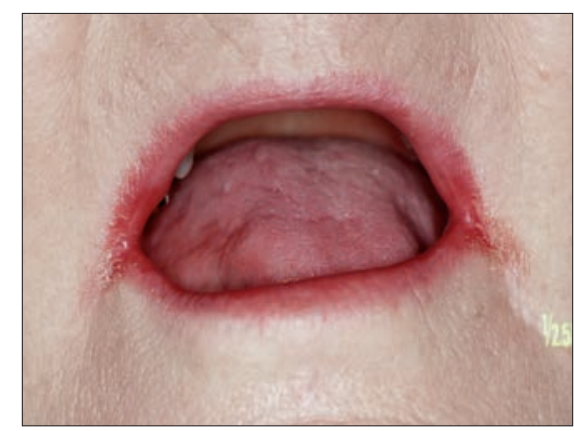

$\triangle$ Fig. 11 :

Chéilite candidosique bilatérale favorisée par la perte de la dimension verticale (coll. Gauzeran).
Il se crée un affaissement du pli commissural qui favorise la macération liée aux suintements d'une salive acide, milieu favorable aux candidoses et aux surinfections bactériennes. La perlèche est souvent assortie d'une autre localisation candidosique buccale, en particulier sous-prothétique.

Une carence vitaminique ou une anémie ferriprive, fréquemment associées, seront recherchées et traitées.

\section{Ulcérations traumatiques prothétiques [5] et dentaires}

Elles sont fréquentes et en rapport avec des prothèses anciennes, des crochets inadaptés ou le traumatisme d'une dent délabrée. La vigilance s'impose car la douleur n'est que rarement verbalisée par le patient $[13,14]$. Les ulcérations d'origine traumatique doivent disparaître rapidement ( 8 à 15 jours) après suppression de la cause irritative. Si tel n'est pas le cas, une biopsie s'impose (fig 12).

Ces lésions ne doivent jamais être négligées. En effet, elles induisent une inflammation chronique qui est susceptible, dans certaines conditions, d'être un
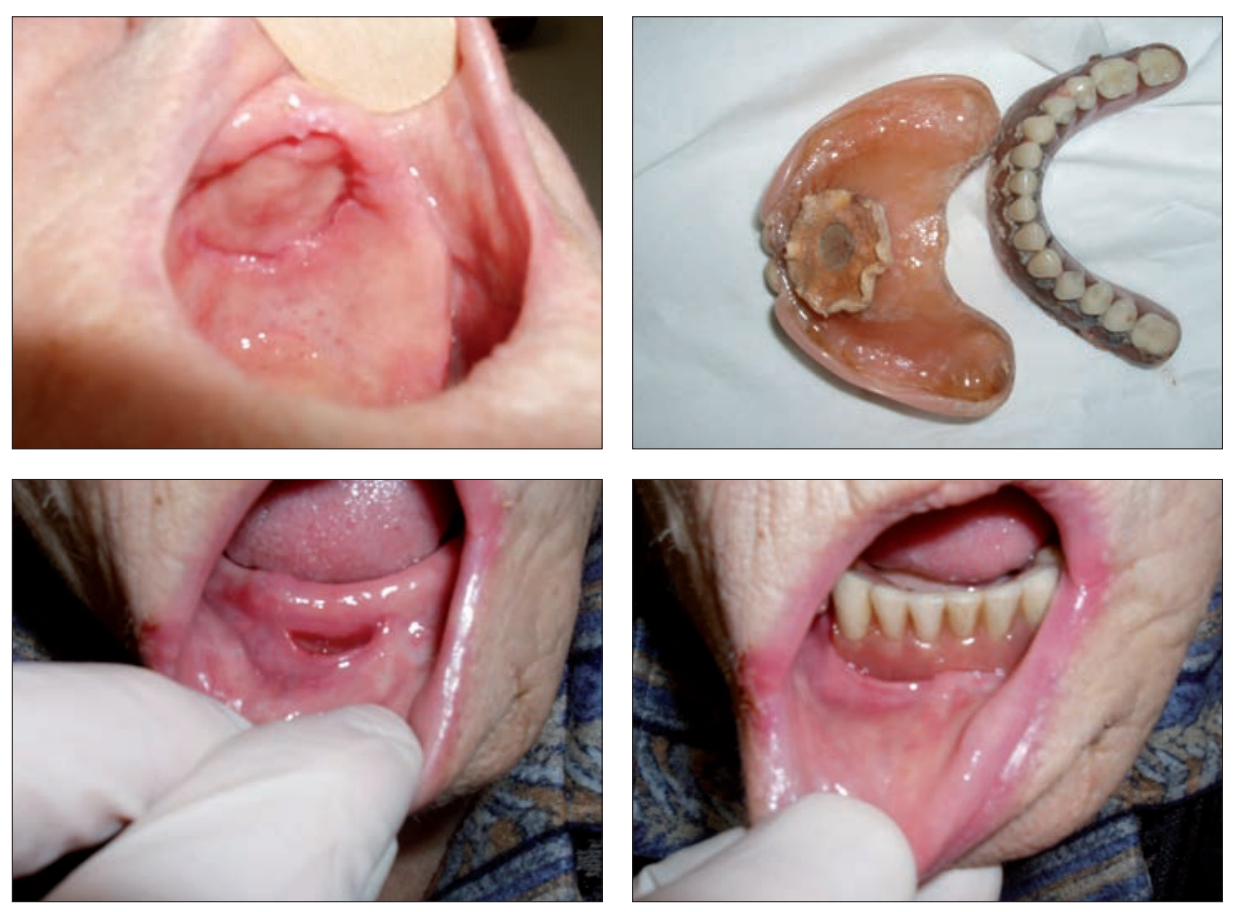

$\triangle$ Fig. 12 a et b :

Ulcération de la muqueuse palatine en rapport avec une prothèse maxillaire ancienne, jamais contrôlée, comportant un dispositif de rétention prothétique de type "ventouse": il existe une communication bucco-nasale (découverte fortuite lors de l'examen clinique) (coll. Saricassapian).

4 Fig. 12 c et d :

Ulcération de la muqueuse vestibulaire inférieure en regard du rebord prothétique antérieur traumatisant (coll. Saricassapian). 


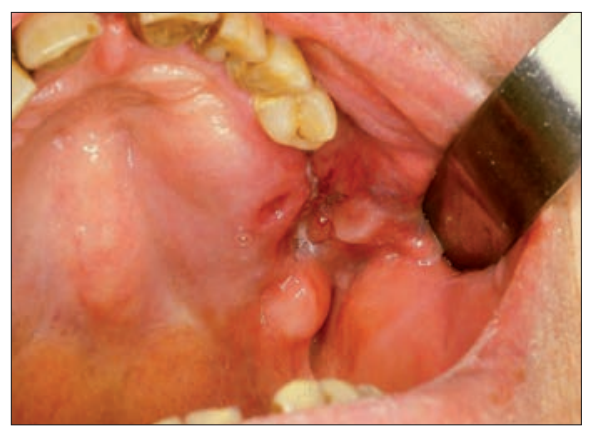

4 Fig. 13:

Ulcération chronique d'origine traumatique liée à une prothèse amovible inadaptée, chez une femme de 68 ans en institution. Néoplasie intraépithéliale (OIN) de bas grade (coll. Gauzeran).

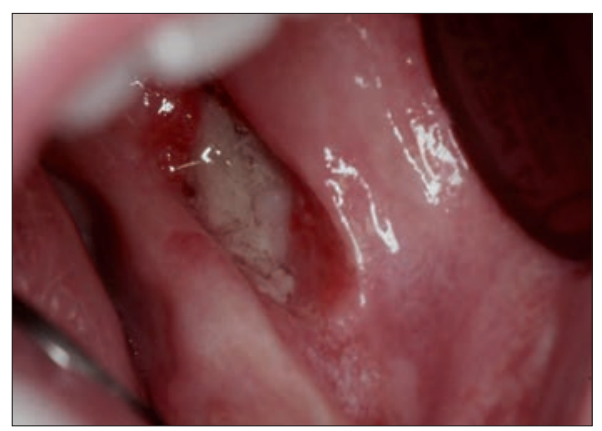

$\triangleleft$ Fig. 14 :

Carcinome

épidermoïde, avec atteinte mandibulaire, sous une prothèse amovible ancienne et inadaptée, chez une femme de 88 ans en institution. Sans facteur de risque associé (coll. Gauzeran). activateur du processus de cancérisation, en particulier chez la personne âgée [15] (fig 13 et 14).

\section{Kératoses réactionnelles}

Les traumatismes chroniques prothétiques ou dentaires sont à l'origine d'ulcérations, mais aussi de réaction kératosique. Les kératoses sont souvent inhomogènes :

I verruqueuses, de couleur blanc nacré, de consistance ferme et au relief plus ou moins prononcé ;

॥ mixtes, associant des zones érosives au sein d'une formation kératosique.

Ces lésions doivent être prises en charge avec la plus grande attention car ce sont des lésions à risque potentiel de transformation maligne, surtout si des facteurs de risque s'ajoutent (tabac, alcool...).

Un examen des aires ganglionnaires cervicales complètera l'examen clinique. La recherche d'une éventuelle adénopathie fait partie intégrante de toute démarche diagnostique. Les lésions persistantes, après suppression de la cause, doivent être biopsiées avant de bénéficier d'une exérèse selon des principes carcinologiques. (fig 15-16 et 17).

\section{LÉSIONS BUCCALES DE MALADIES DERMATOLOGIQUES $[8,11]$}

Elles concernent essentiellement, chez la personne âgée, les dermatoses bulleuses et le lichen plan.

\section{Dermatoses bulleuses}

Parmi ces lésions auto-immunes, à décollement sous-épithélial, la pemphigoïde bulleuse et la pemphigoïde cicatricielle sont plus fréquentes chez le sujet âgé. Elles rentrent dans le cadre des gingivites érosives chroniques.

Les gencives sont très érythémateuses, luisantes et douloureuses au contact. Après léger frottement de la gencive, il est possible de décoller, au moyen d'une précelle, l'épithélium : c'est le signe de la pince, dans ce cas positif (fig 18 et 19).

\section{Lichen plan $[5,16,17]$}

D'origine vraisemblablement dysimmunitaire, le lichen plan buccal est une maladie cutanéomuqueuse

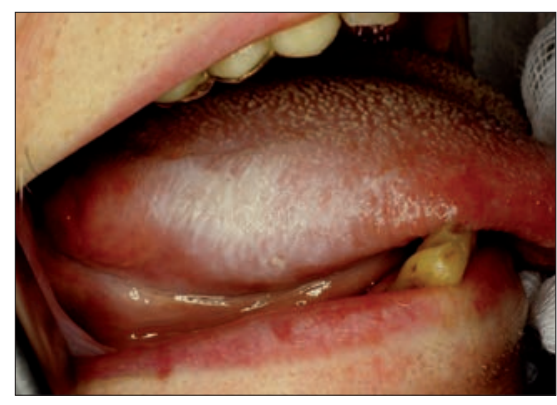

$\triangle$ Fig. 15 :

Kératose réactionnelle homogène bénigne liée à une irritation chronique d'origine dentaire (coll. Gauzeran).

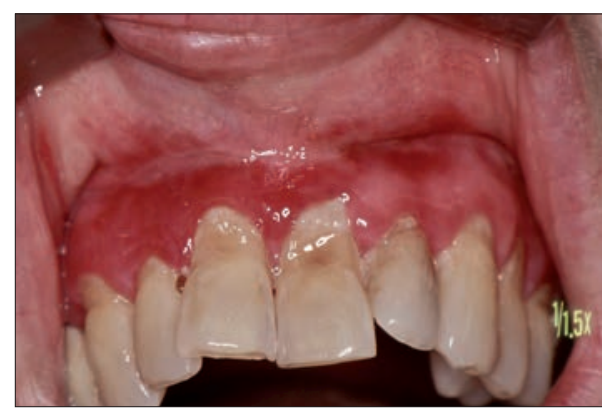

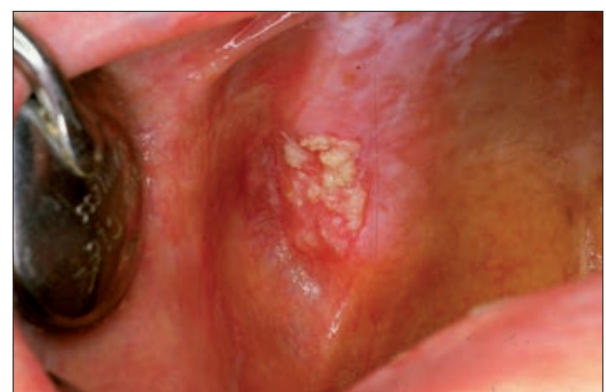

$\triangle$ Fig. 16:

Kératose réactionnelle inhomogène bénigne, liée à une irritation chronique par une prothèse amovible inadaptée, chez un homme de 75 ans (coll. Gauzeran).

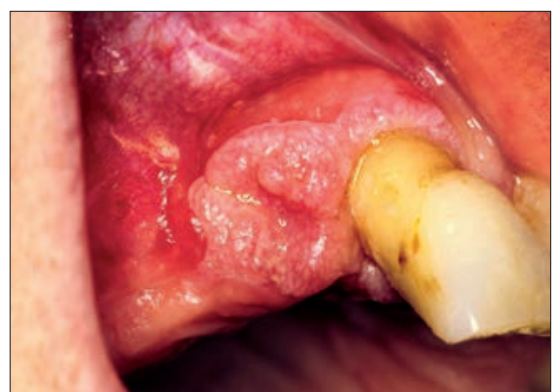

$\triangle$ Fig. 17 :

Kératose inhomogène d'origine traumatique sous une prothèse amovible ancienne. Néoplasie intra-épithéliale de bas grade (coll. Gauzeran).
4 Fig. 18 :

Pemphigoïde

cicatricielle (coll.

Gauzeran).

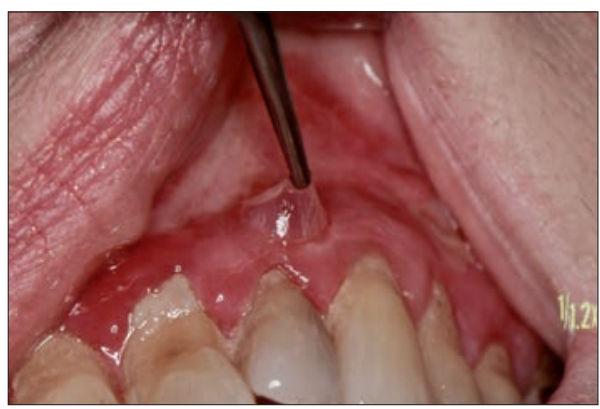

$\triangleleft$ Fig. 19:

Signe de la pince

positif (coll.

Gauzeran). 


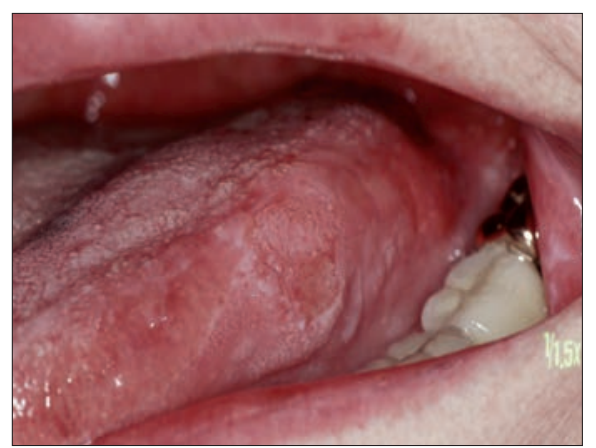

$\triangle$ Fig. 20 :

Carcinome épidermoïde sur lichen plan ancien chez une femme de 70 ans. Pas de facteur de risque associé (coll. Gauzeran)

inflammatoire chronique. La localisation buccale est plus fréquente que le lichen cutané.

Cette dermatose évolue sur plusieurs années pour aboutir à un état cicatriciel dit : état post-lichénien.

Durant cette évolution, le lichen subit une succession de poussées plus ou moins inflammatoires et change d'aspect pour devenir atrophique ou scléro-atrophique.

Souvent des ulcérations apparaissent et cicatrisent mal. Parfois, le lichen se présente sous forme de placards hyperkératosiques épais et rugueux avec coexistence possible de zones atrophiques et/ou érosives.

Le lichen au stade post-lichénien est une lésion à risque potentiel de transformation maligne. C'est dire que la surveillance doit être rigoureuse et fréquente car la cancérisation est observée dans 2 à $5 \%$ des cas selon les auteurs. Au moindre doute, une biopsie ou des biopsies multifocales, en fonction de l'aspect clinique, doivent être pratiquée (fig 20).

\section{CARCINOMES ÉPIDERMOÏDES (CE) $[8,11,18]$}

Ils représentent $90 \%$ des cancers des voies aérodigestives supérieures dont environ $40 \%$ atteignent la muqueuse buccale.

Ces cancers touchent surtout une population entre 40 et 65 ans qui présente le facteur de risque essentiel qu'est la double intoxication tabac-alcool [11].

Néanmoins, les personnes âgées peuvent développer un carcinome épidermoïde sans ce facteur de risque [5]. Il est d'ailleurs noté un second pic de fréquence entre 70 et 80 ans.

D'après une étude de l'Institut Curie, 3 \% des malades traités entre 1970 et 1995 , pour un cancer des VADS sont âgés de 80 ans et plus [19].

Le taux de non-fumeurs dans cette population est de $17 \%$ ( $1,5 \%$ tous âges confondus) et la consommation d'alcool supérieure à $40 \mathrm{~g} / \mathrm{j}$ est de $32 \%$ (92 \% tous âges confondus).

Cette étude montre une fréquence des localisations buccales de $46 \%$ pour les plus de 80 ans avec une prédilection pour la langue mobile, la face interne des joues et la gencive.

Pour d'autres auteurs, les carcinomes intéressent également tous les sites de la cavité buccale [20].

Les mêmes formes cliniques sont retrouvées quelle que soit la tranche d'âge : verruqueuses, ulcéreuses, ulcéro-végétantes, ulcéro-infiltrantes... (fig 21 à 25). La détection précoce de cette redoutable pathologie permet la mise en route d'un traitement adapté au

Localisations linguales de carcinome épidermoïde sans facteur de risque associé

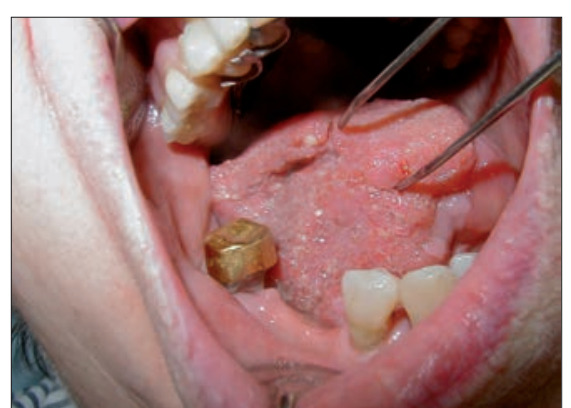

$\triangle$ Fig. 21 :

T4 N1 M0 forme ulcéro-infiltrante chez une femme de 81 ans (coll. Saricassapian).

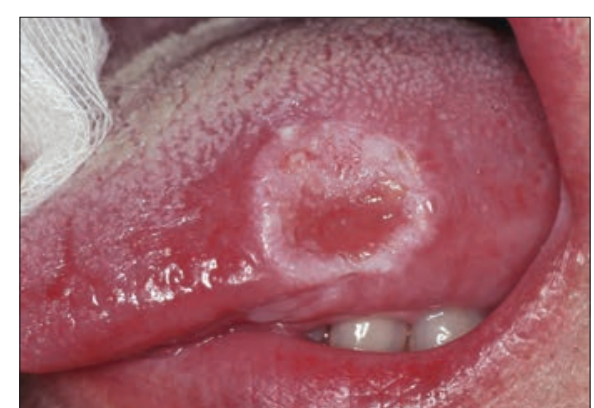

$\triangle$ Fig. 22 :

T1 N0 M0 forme ulcéreuse chez une femme de 75 ans (coll. Gauzeran).

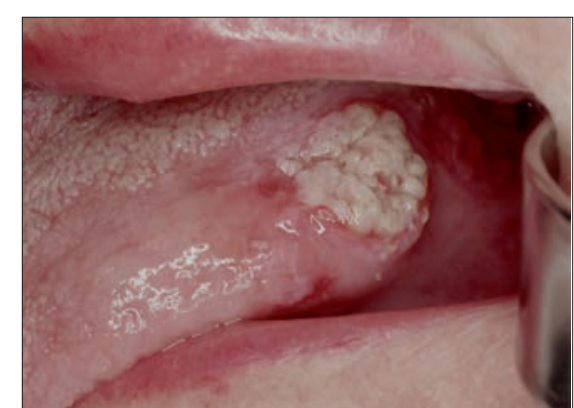

$\triangle$ Fig. 23 :

T2 N1 M0 forme verruqueuse chez une femme de 72 ans (coll. Gauzeran).

Localisations gingivales de carcinome épidermoïde sans facteur de risque associé

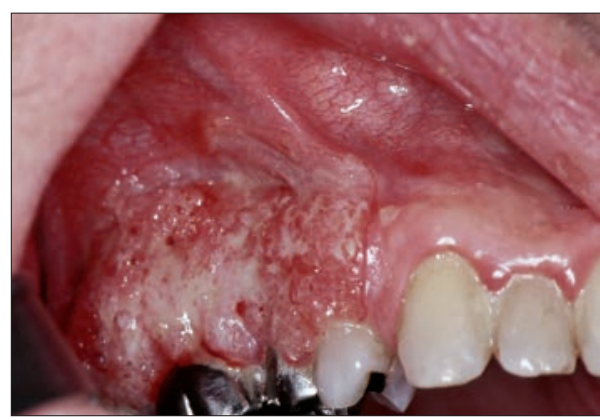

$\triangleleft$ Fig. 24 :

T4 N1 M0 (forme ulcéro-végétante) chez une femme de 70 ans (coll. Gauzeran).

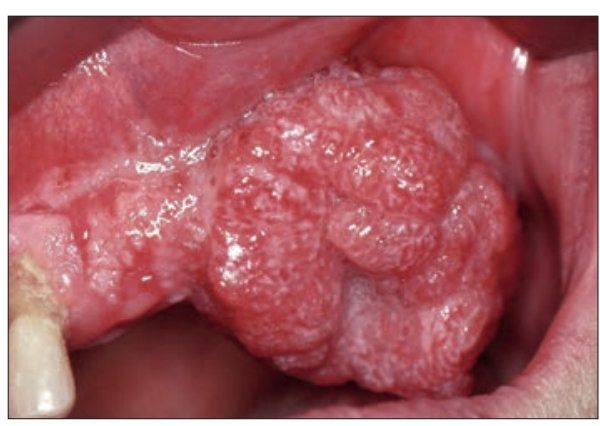

4 Fig. 25 :

T3 N0 M0 (forme bourgeonnante) chez une femme de 85 ans (coll. Gauzeran). 
cas par cas, tout en respectant un meilleur confort de vie, avec un pronostic plus favorable du fait du faible pouvoir évolutif de ces lésions chez le sujet âgé [20].

\section{PATHOLOGIES DE LA LANGUE $[5,18]$}

Certaines d'entre elles sont très fréquentes chez le vieillard. Elles peuvent être le reflet d'une pathologie générale sous-jacente.

\section{Langue plicaturée (fissurée, scrotale) [21]}

Très fréquemment observée et asymptomatique, hormis une sensibilité exacerbée aux épices, à l'alcool et aux aliments trop chauds ou acides, elle n'est pas franchement pathologique mais peut inquiéter par son aspect : la face dorsale de la langue présente de multiples fissures et crevasses surtout dans le sens antéropostérieur. Il n'y a pas d'étiologie retrouvée et pas de traitement à proposer (fig 26).

\section{Glossite exfoliatrice migratrice}

La glossite exfoliatrice migratrice (langue géographique, exfoliato areata linguae, areata migrans, glossite exfoliatrice marginée, glossite dépapillante...) est une pathologie d'étiologie inconnue. Il s'agit d'une exfoliation des papilles filiformes par plaques, au contour net, de couleur blanc jaunâtre, inconstante $\mathrm{du}$ fait de la repousse irrégulière des papilles.

La lésion peut durer de quelques semaines à plusieurs années. Certains auteurs la considèrent comme une manifestation psychosomatique, d'autres comme la manifestation isolée d'un psoriasis (confirmé par l'examen histologique) [22] (fig. 27-28).

\section{Glossite atrophique}

La glossite atrophique (langue rouge dépapillée) [23] est un état inflammatoire dont la manifestation clinique se caractérise par une dépapillation complète de la langue qui présente un aspect lisse et carminé.

Compte tenu du contexte inflammatoire marqué, le patient peut se plaindre de douleurs au contact de certains aliments.

Une carence en vitamines du groupe B ainsi qu'une anémie ferriprive sont à rechercher.

Elle peut être associée à une hyposialie sévère et à une perlèche bilatérale (fig 29).

\section{Paresthésies buccales médicalement inexpliquées}

(Paresthésies buccales psychogènes, stomatodynies, glossodynies [23].)

Il s'agit essentiellement de sensations anormales de brûlures, de piqûres au niveau de la langue avec parfois d'autres localisations buccales.

Elles ne sont expliquées par aucune anomalie locale cliniquement décelable.
Ces douleurs, d'origine psychique très probable, sont la somatisation d'un état dépressif sous-jacent.

Il est impératif de ne pas sous-estimer la souffrance du patient, de rester à son écoute, de s'interdire la prescription de traitements locaux irritants et, surtout, de réaliser un examen minutieux de la cavité buccale afin d'éviter tout diagnostic « de facilité » en passant à côté d'une lésion qui serait à l'origine de ces symptômes. Certains auteurs préconisent la prescription de Tégrétol $^{\circledR}$, de Laroxyl ${ }^{\circledR}$ ou un traitement antidépresseur, en collaboration avec le médecin traitant.

Une carence en vitamine B12 sera recherchée [37].

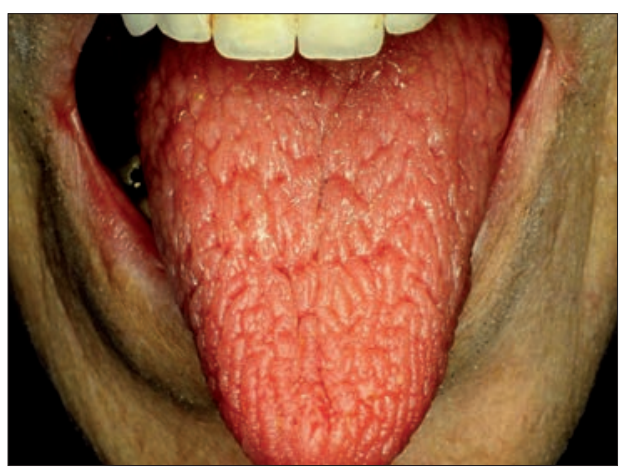

4 Fig. 26 :

Langue fissurée

(coll. Gauzeran).

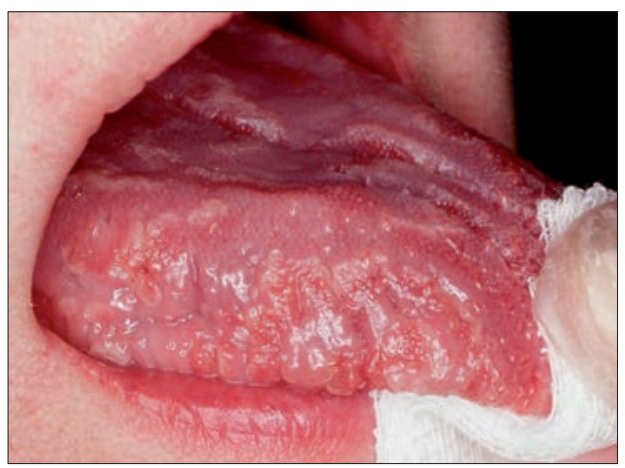

4 Fig. 27:

Glossite exfoliatrice migratrice (coll. Gauzeran).

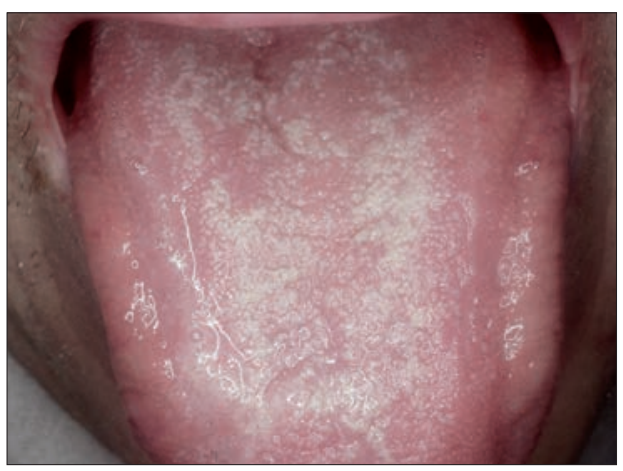

4 Fig. 28 :

Psoriasis lingual (coll. Gauzeran).

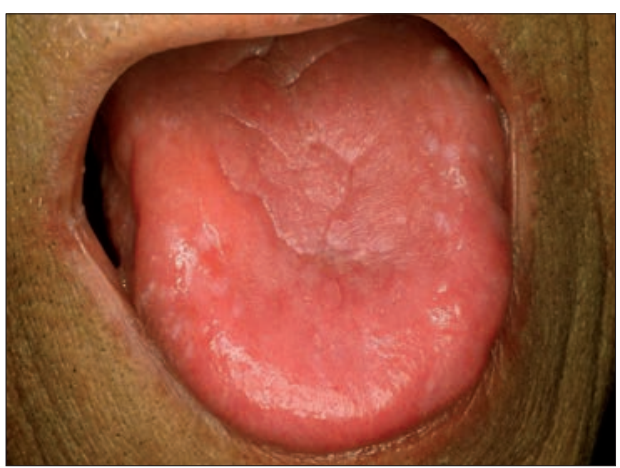

4 Fig. 29 :

Glossite atrophique (coll. Gauzeran). 


\section{OSTÉONÉCROSES EN RAPPORT AVEC LES BIPHOSPHONATES $[18,24$,}

$25,26,27]$

Les ostéonécroses liées aux biphosphonates sont de plus en plus fréquemment observées chez les personnes âgées.

On peut diviser ces médicaments en deux groupes : v ceux utilisés essentiellement dans le traitement des ostéolyses malignes (métastases osseuses des cancers du sein, de la prostate...), de l'hypercalcémie maligne... Le rôle de ces molécules (acides pamidronique et zolédronique) administrées par voie parentérale, dans les cas d'ostéonécrose des maxillaires, ne fait aucun doute aujourd'hui (incidence proche de $1 \%$ avec l'acide zolédronique);

r ceux utilisés dans le traitement des pathologies non oncologiques (ostéoporose post-ménopausique et cortisonique), administrés en per os (Bonviva ${ }^{\oplus}$, Fosamax $^{\oplus}$, Didronel $^{\oplus}$, Actonel $^{\oplus}$, Aclasta $\left.^{\circledR} . ..\right)$.

D'après une étude américaine, $27 \%$ des femmes de 80 ans présenteraient une ostéopénie et $70 \%$ une ostéoporose. Les biphosphonates réduiraient d'environ 20 à $50 \%$ le risque de fracture pathologique.

L'apparition d'ostéonécrose chez ces patients reste encore très controversée : l'incidence de cet effet indésirable dans cette indication n'est pas connue et les facteurs de risque sont encore incertains.

Ainsi, toutes les recommandations incitent à une attitude prophylactique afin d'en limiter la survenue. Les lésions se présentent sous la forme d'une exposition osseuse persistante dans la cavité buccale, le plus souvent consécutive à un geste chirurgical (avulsion...), à un traumatisme prothétique... mais elles peuvent aussi se manifester spontanément. Une surinfection bactérienne peut y être associée.

L'indication d'un acte chirurgical bucco-dentaire chez un patient bénéficiant d'un traitement par biphosphonates doit être soigneusement pesée (rapport bénéfice/ risque) en collaboration avec le médecin traitant. Le patient (ou sa tutelle) doit être bien informé des risques et son consentement doit être acquis.

Les éléments à prendre en compte sont le type de molécule utilisée, la durée du traitement, les doses administrées, les traitements médicamenteux associés, les comorbidités et l'hygiène bucco-dentaire (fig 30, $31,32)$.

\section{ULCÉRATIONS BUCCALES PAR IRRITATION MÉDICAMENTEUSE [28]}

Bien que rares, nous pouvons les observer chez les patients âgés à l'état général dégradé : troubles de la déglutition, déficit cognitif sévère, déshydratation, état grabataire...

Tout médicament sous forme de comprimé est potentiellement responsable d'une ulcération de la muqueuse buccale par irritation mécanique et/ou chimique par la stagnation prolongée sur un même site (muqueuse jugale, sillon gingivo-jugal, pelvi-lingual...) dans un contexte d'hyposialie et de septicité buccale.

Les complications infectieuses loco-régionales constituent généralement le motif de la consultation émanant du personnel soignant de l'institution.

D'autre part, les gels à usage local contenant de l'acide salicylique et de l'alcool, en application excessive
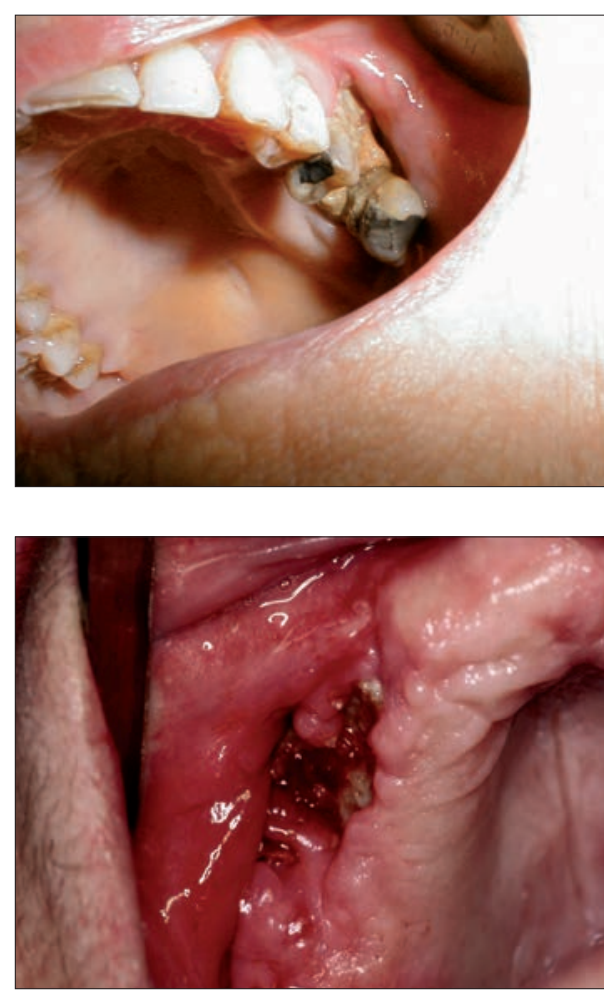

$\triangleleft$ Fig. 30 a et b :

Ostéochimionécrose suite à l'avulsion de 26, compliquée d'une sinusite maxillaire gauche chez une patiente présentant des métastases osseuses d'un cancer du sein : 8 cures d'acide zolédronique et 4 cures d'acide pamidronique avaient été administrées par voie parentérale (coll. Saricassapian). $\triangleleft$ Fig. 31 :

Ostéochimionécrose spontanée par biphosphonates par voie parentérale (acide zolédronique), chez un malade présentant des métastases vertébrales d'un adénocarcinome prostatique (coll. Gauzeran).

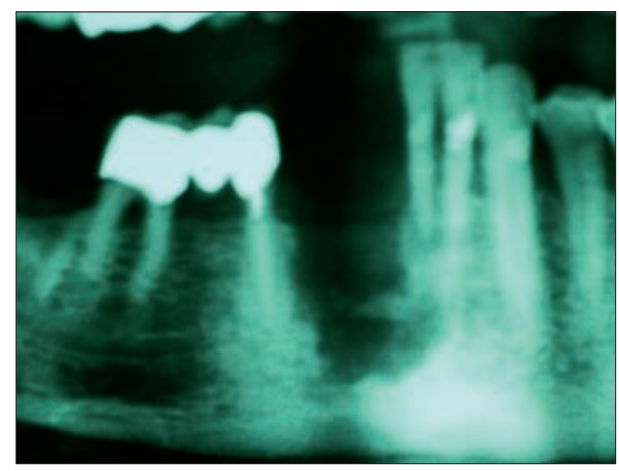

$\triangleleft$ Fig. 32 :

Ostéochimionécrose mandibulaire spontanée par biphosphonates per os (acide risédronique), chez une femme de 75 ans traitée pour une ostéoporose (coll. Gauzeran). 

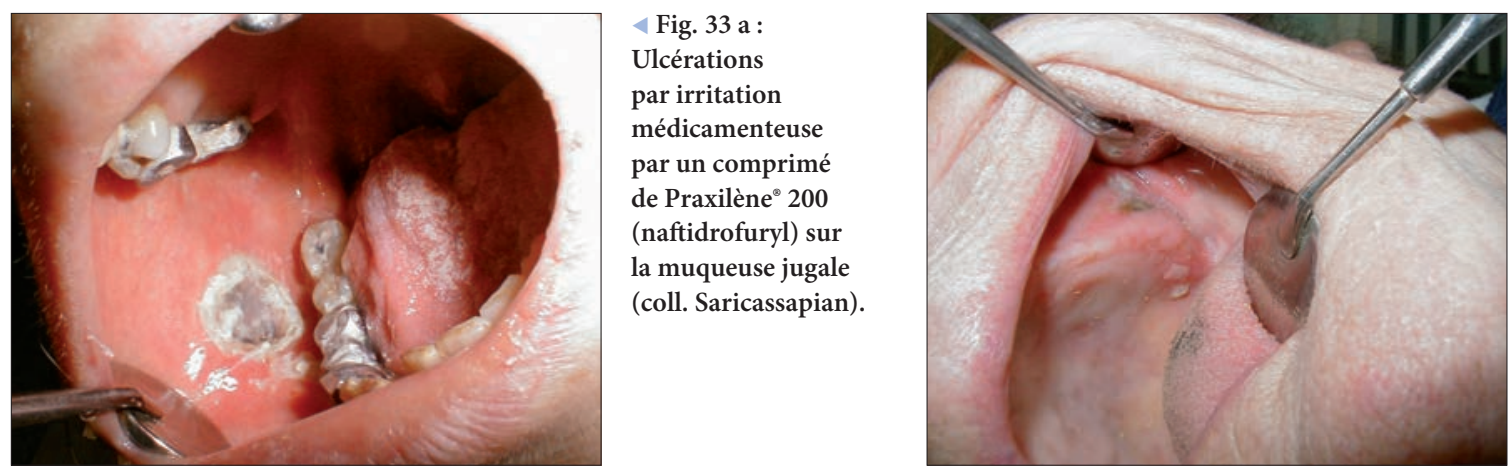

4 Fig. 33 b :

Ulcérations

par irritation médicamenteuse par un comprimé de Tardyféron (sulfate ferreux) au fond du vestibule supérieur gauche (coll. Saricassapian).

(sous la prothèse dentaire par exemple) peuvent être également à l'origine d'ulcérations.

Une antibiothérapie adaptée en cas de surinfection et les soins locaux permettent une guérison rapide de la lésion (fig 33).

\section{CONCLUSION $[2,3,29,30]$}

Compte tenu des pathologies variées, fréquentes et parfois atypiques de la muqueuse buccale des personnes âgées, il n'est guère besoin d'insister sur la nécessité de la mise en place d'une politique de prévention globale destinée aux résidents des institutions. En effet, la plupart d'entre eux sont en dehors du circuit des soins depuis plusieurs années.
Certaines pathologies peuvent avoir une évolution loco-régionale pouvant engager le pronostic vital, d'autres favoriser une altération rapide de l'état général. Par conséquent, nous insistons sur l'absolue nécessité d'un dépistage bucco-dentaire systématique pour les résidents entrant en EHPAD.

Un article de 2003 dans La Lettre de l'Ordre national des chirurgiens-dentistes consacré à l'état bucco-dentaire des personnes âgées dépendantes et intitulé " Des bouches à l'abandon", reste malheureusement d'actualité [31].

Cependant, La Lettre publiée en mai 2012 faisant état d'un futur Rapport parlementaire sur la prévention, nous permet d'espérer... [32].

Quoi qu'il en soit, notre rôle en tant qu'odontologiste est de rester particulièrement attentif et vigilant vis-àvis de ces patients vulnérables.

\section{Bibliographie}

[1] Agence française de sécurité sanitaire des produits de santé. Prévenir la iatrogénèse médicamenteuse chez le sujet âgé. Afssaps juin 2005

[2] Ettiger RL, Beck JD. Geriatric dental curriculum and the needs of the elderly. Spec Care Dentist 1984;4(5):207-13.

[3] Folliguet M. et al. Prévention bucco-dentaire chez les personnes âgées. Direction Générale de la Santé, SD2B mai 2006.

[4] Dorin M. et al. Etat de santé bucco-dentaire des personnes âgées en établissement hébergeant des personnes âgées dans l'Essonne. Inf Dent 2003;23:1547-1556.

[5] Gauzeran D. Pathologie des muqueuses orales de la personne âgée. Inf Dent 1993;75(35):2643-2653.

[6] Ragot JP, Auriol M, Bertrand JC. Vieillissement des glandes salivaires. Actual Odonto Stomatol 2001;215:345-366.
[7] Mauprivez C. Mieux prescrire chez le sujet âgé. Inf Dent 2011;93(32):65-76.

[8] Kuffer R, Lombardi T, Husson-Bui C, Courrier B, Samson J. La muqueuse buccale : de la clinique au traitement. Paris : Ed. Med'com 2009.

[9] Gauzeran D. Candidoses buccales. Paris : Ed. AC3P-Pred, 2000.

[10] Poidatz E. Pathologie buccale du sujet âgé. Chir Dent Fr 1991:61(555):35-38

[11] Gauzeran D. Lésions à risque et cancers de la bouche. Paris : Ed. CdP ; Wolters Kluwer France 2007.

[12] Courrier B, Husson C. Pathologie de la muqueuse buccale chez la personne âgée. Rev Odonto Stomatologie 1998;27(1):49-54.

[13] Wary B. Douleurs chez les personnes âgées. Douleurs 2002;3(5):207-209.

[14] Lapeer GL. Dementia's impact on pain sensation: a serious clinical dilemma for dental geriatric care givers. J Can Dent Assoc 1998;64(3):182-192.
[15] Gauzeran D. Traumatismes prothétiques chroniques, personnes âgées et carcinome. Alternatives 2000;14:11-16.

[16] Le Charpentier Y, Auriol M. Histopathologie bucco-dentaire et maxillofaciale. Paris : Masson, 1997.

[17] Samson J, Carrel JP. Pathologie de la muqueuse buccale chez la personne âgée. Alpha Oméga News 2003;81.

[18] Feki A, Abi Najm S, Descroix V, Gauzeran D, Gourmet R, Guichard M, Kleinfinger S, Lescaille G, Lombardi T, Samson J. Le chirurgien-dentiste face au cancer. Les dossiers de l'ADF. Paris : Edition ADF, 2008.

[19] Jouffroy T, et al. Cancers des voies aérodigestives supérieures chez le sujet de $\mathbf{8 0}$ ans et plus. Rapport du $31^{\mathrm{e}}$ congrès de la Société Française de Carcinologie CervicoFaciale 1998:67-73.

[20] Gaillard-Perera H, Gaillard A. Les carcinomes de la cavité buccale indépendants du tabac et des boissons alcooliques. Rev Stomatol Chir Maxillofac 1992;93(1):58-59. 
[21] Beaven DW, Brooks SE. Atlas en couleurs de diagnostics cliniques de la langue. Paris : Maloine, 1989.

[22] Guilhou JJ. Psoriasis : aspects cliniques et diagnostic différentiel. EMC Dermatologie 1992;12310 A10.

[23] Loup A, Lombardi T, Samson J. Carence en vitamine $B 12$ faisant évoquer des paresthésies buccales médicalement inexpliquées. Méd Buccale Chir Buccale 2011;17(1):69-70.

[24] Abi Najm S, et al. Ostéonécrose des maxillaires dues aux biphosphonates ( $1^{\mathrm{re}}$ partie) : mise au point. Med Buccale Chir Buccale 2008;14(1):5-18.
[25] Abi Najm S, et al. Ostéonécrose des maxillaires dues aux biphosphonates (2 ${ }^{\mathrm{e}}$ partie) : expérience genevoise. Med Buccale Chir Buccale 2008;14:2,63-84.

[26] Académie Nationale de Chirurgie Dentaire. Biphosphonates et odontologie : attitude du chirurgien-dentiste. Bulletin 2009;52.

[27] Agence française de sécurité sanitaire des produits de santé. Recommandations sur la prise en charge bucco-dentaire des patients traités par biphosphonates. Afssaps décembre 2007.

[28] Sarry B. Ulcérations buccales par irritation médicamenteuse chez la personne âgée. Congrès de la SFMbCb, Dijon 2007.
[29] Koopman C, Coulthard S. The Oral cavity and aging. Otolaryngol Clin North Am 1982;15(2):293-312.

[30] Fries BE, et al. Accelerate dysfunction among the very oldest-old in nursing homes. J Gerontol Biol Sci Med Sci 2000;55(6):M336-41.

[31] Ordre National des Chirurgiens-Dentistes. Personnes âgées dépendantes : des bouches à l'abandon. La Lettre de l'ONCD 2003;15:18.

[32] Ordre National des Chirurgien-Dentistes. Un rapport parlementaire en faveur de la prévention. La Lettre de l'ONCD mai 2012;107:21.

\section{Analyse}

\section{François Descamps}

\section{Pratique de l'empreinte en prothèse fixée}

Du pilier naturel à l'implant, des techniques classiques à la CFAO. Éditions CdP, Rueil-Malmaison, 2012.

L'empreinte est une étape incompressible de la réalisation d'une prothèse fixée. Qu'il s'agisse d'une empreinte physique conventionnelle ou d'une empreinte optique dématérialisée, les critères de validation clinique initiale et le cahier des charges à respecter restent garants de la réussite du binôme clinicien-prothésiste. La valeur de cet enregistrement conditionne la fabrication et la fiabilité de l'élément prothétique final, et donc son intégration biologique.

Très contemporain dans son analyse de l'avenir numérique des empreintes en prothèse fixée, ce guide clinique s'ouvre vers l'avenir tout en restant proche des réalités quotidiennes. L'auteur détaille efficacement les différentes étapes et objectifs de chaque technique d'empreinte en six chapitres. Sont identifiés, dans un premier temps, les paramètres à prendre en compte dans la réalisation de l'empreinte et les problèmes qu'ils soulèvent. Les risques d'imprécision sont mis en évidence dans chaque maillon de la chaîne de fabrication. Plusieurs moyens de prévention de ces risques et les conditions de la réussite (porte-empreinte, matériaux adaptés, accès à la limite cervicale et au sulcus) sont présentés à l'appui d'une grande expérience clinique de l'empreinte dite « conventionnelle » sur pilier naturel ou implantaire (spécificité des structures à enregistrer, paramètres et contraintes des matériaux).

Les esprits avant-gardistes sont pour une fois satisfaits ou en tout cas alléchés car l'empreinte optique fait enfin son apparition dans un ouvrage généraliste de prothèse fixée. L'évolution technologique de la prothèse fixée, qu'il s'agisse des solutions qu'elle apporte (prothèses implantoportées, restaurations tout céramique) ou des processus de fabrication qu'elle emprunte (CFAO), transforme la conception classique de la prise d'empreinte et sa représentation dans l'esprit du clinicien. Mais convient-il pour

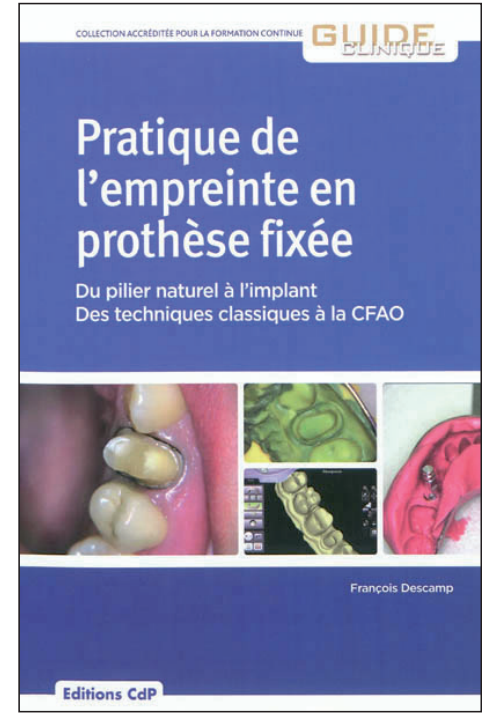
autant de s'habituer à une empreinte dématérialisée supportée, non plus par un porte-empreinte, mais par un support numérique ? Non, pas encore et pas avant quelques années selon François Descamps. Sa conclusion reste réaliste et prudente : il faut avancer avec son temps mais sûrement. L'auteur invite avec raison à relativiser toute prédiction technologique dans la spécialité odontologique, particulièrement en France où la politique médico-sociale dentaire n'a jamais été tournée ni vers la reconnaissance des techniques de pointe, ni vers une discrimination qualitative de l'acte pratiqué.

Fervents défenseurs de l'empreinte optique d'une part et nombreux conservateurs de techniques actuelles connues, rassurantes et maîtrisées d'autre part s'accorderont en tout cas pour reconnaître que le travail de synthèse fourni par François Descamps mérite d'être parcouru avec attention. Ce volume réalise le tour d'horizon le plus complet possible de la thématique de la pratique de l'empreinte en prothèse fixée.

Amandine PARA 